\title{
La nécropole à enclos de l'âge du Fer des Truberdières, à Écommoy (Sarthe)
}

The Iron Age cemetery of Les Truberdières, in Écommoy (Sarthe)

Die eisenzeitliche Nekropole von Les Truberdières bei Écommoy (Sarthe)

La necrópolis de la Edad del Hierro de Les Truberdières, en Écommoy (Sarthe)

Anne-Louise Hamon, Axel Levillayer, Isabelle Le Goff et Catherine Moreau

\section{OpenEdition}

Édition électronique

URL : https://journals.openedition.org/rao/6633

DOI : $10.4000 /$ rao.6633

ISSN : $1775-3732$

Éditeur

Presses universitaires de Rennes

Édition imprimée

Date de publication : 21 décembre 2021

Pagination : 125-138

ISBN : 978-2-7535-8668-0

ISSN : 0767-709X

\section{Référence électronique}

Anne-Louise Hamon, Axel Levillayer, Isabelle Le Goff et Catherine Moreau, « La nécropole à enclos de l'âge du Fer des Truberdières, à Écommoy (Sarthe) », Revue archéologique de l'Ouest [En ligne], 37 | 2021, mis en ligne le 21 décembre 2021, consulté le 11 octobre 2022. URL : http:// journals.openedition.org/rao/6633; DOI : https://doi.org/10.4000/rao.6633 


\title{
La nécropole à enclos de l'âge du Fer des Truberdières, à Écommoy (Sarthe)
}

\author{
The Iron Age cemetery of Les Truberdières, in Écommoy (Sarthe)
}

Anne-Louise Hamon

Responsable d'opérations, Inrap Grand-Ouest (anne-louise.hamon@inrap.fr)

Axel Levillayer

Responsable d'opérations, Grand Patrimoine de Loire-Atlantique, Pôle archéologie préventive et programmée, chercheur associé à l'UMR 6566 CReAAH (axel.

levillayer@loire-atlantique.fr)

Isabelle Le Goff

Anthropologue, Inrap Centre - Île de France, chercheure associée à l'UMR 7206 Éco-Anthropologie et Ethnobiologie (isabelle.le-goff@inrap.fr)

Catherine Moreau

Ingénieure d'études, Service régional de l'archéologie Pays de la Loire, chercheure associée à l'UMR 6566 CReAAH (catherine.moreau@culture.gouv.fr)

Résumé : La nécropole à enclos des Truberdières à Écommoy, située dans le sud du département de la Sarthe, a été fouillée en 2001. Une série de quinze petits cercles fossoyés et deux doubles enclos quadrangulaires, datés de la transition entre le premier et le second âge du Fer y constitue l'un des sites funéraires les plus denses mis au jour en Pays de la Loire pour cette période. Un mobilier céramique et métallique ténu contribue à en souligner le caractère remarquable en s'insérant au sein de la zone d'influence culturelle du Bassin parisien et de l'Aisne-Marne.

Abstract: The monumental cemetery excavated in 2001 at Les Truberdières, Ecommoy, is located in the south of the Sarthe. Fifteen circular ditches and two double quadrangular enclosures, dating to the transition between the Early and Late Iron Age constitutes one of the densest burial sites discovered in the Pays de la Loire for this period. The few pottery and metal artefacts underline its remarkable character as part of the cultural influence of the Parisian Basin and the Aisne-Marne region.

Mots clés: nécropole, âge du Fer, enclos circulaire, enclos quadrangulaire, incinération.

Keywords: burial ground, Iron Age, circular ditches, quadrangular enclosures, cremation.

C'est à la suite de deux interventions de diagnostic et d'évaluation réalisées sur la future zone d'activité des Truberdières en Écommoy (Sarthe) en 2000 et 2001 par Stéphane Vacher (Inrap) et Anne-Louise Hamon (Inrap), que le site des Truberdières a fait l'objet en septembre 2001 d'un décapage mécanique sur $3000 \mathrm{~m}^{2}$ suivi de la fouille manuelle des structures en creux mises au jour. Aucun autre indice en lien avec le site funéraire n'a été découvert au cours des sondages préalables, à proximité de l'emprise fouillée. L'essentiel de la nécropole a été abordé, même s'il est possible que des vestiges figurent au-delà, notamment des limites orientale et méridionale du décapage.

Le site des Truberdières prend place à $2 \mathrm{~km}$ au sudest de la ville d'Écommoy, au sein du paysage vallonné du Bélinois, à $100 \mathrm{~m}$ d'altitude, dans une zone basse au niveau de laquelle s'écoule le petit ruisseau de Beaussay (fig. 1). Il est installé sur un substrat cénomanien dit des sables du Maine, rencontré sur le terrain sous la forme d'un sable meuble de couleur beige à jaune, à graviers et blocs siliceux épars et traces de ferruginisation. Un phénomène pédologique de litage, apparu à partir des 
Figure 1 : Localisation du site d'ÉcommoyLes Truberdières (๑ A.-L. Hamon).

Figure 1: Location of the Ecommoy-Les Truberdières site (○ A.-L. Hamon).

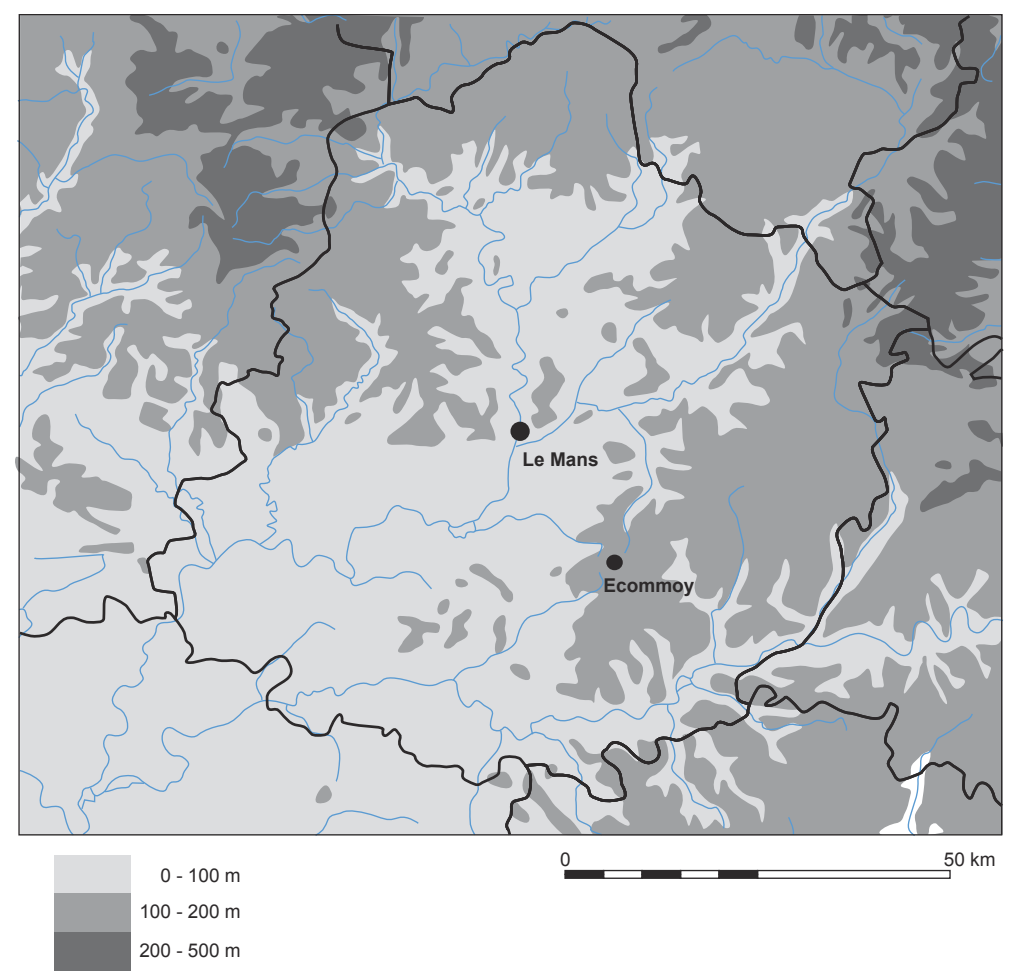

battements de nappes d'eau superficielles, induit l'uniformisation quasi-totale des comblements archéologiques dont les stratigraphies originelles ne sont souvent plus lisibles. Ainsi, le remplissage des excavations apparaît comme remarquablement homogène. Les coupes observées sont toutes ressemblantes, majoritairement composées d'une alternance régulière de niveaux de sable brun-rouille de moins de $3 \mathrm{~cm}$ d'épaisseur et d'horizons sableux orangés épais d'une quinzaine de centimètres.

La nécropole se compose d'une série de quinze petits enclos fossoyés de forme annulaire plus ou moins régulière, de deux doubles enclos quadrangulaires et d'un ensemble de 51 fosses et 15 trous de poteau répartis sur une surface décapée de $3000 \mathrm{~m}^{2}$ (fig. 2). Un seul long fossé linéaire (structure 16/106) figure sur le tiers sud du site, au niveau duquel il rencontre l'un des enclos circulaires (structure 1). Les fragments de poterie et les restes céramiques et métalliques mis au jour dans les fossés, tant sur les enclos quadrangulaires que curvilignes, livrent des datations relativement homogènes et permettent d'attribuer le site à la fin du premier âge du Fer, ou au début de La Tène ancienne. Seule une fibule trouvée parmi les restes osseux de l'incinération 144 témoigne de la perduration du site jusqu'au Iv ${ }^{\mathrm{e}}$ s. av. n. è.

\section{LES ENCLOS ANNULAIRES}

Deux catégories de format peuvent être définies parmi
Les fossés 3, 4, 7, 8, 26, 71, 73, 114 et 115 atteignent entre 3 et $5 \mathrm{~m}$ d'envergure, alors que les creusements 1,2 , 6, 39, 72 et 117 constituent un ensemble de cercles plus imposants, de 5 à $7 \mathrm{~m}$ de diamètre (tabl. 1 et 2).

À la première série correspond une largeur de fossés très variable, parfois sur un même enclos, avec des mesures étendues de $0,3 \mathrm{~m}$ à $0,8 \mathrm{~m}$, la moyenne des largeurs demeurant autour de $0,5 \mathrm{~m}$. La profondeur des creusements atteint moins de $0,3 \mathrm{~m}$, révélant un arasement très prononcé des niveaux archéologiques. Tous sont comblés de sable plus ou moins uniforme, de couleur variant du beige au brun foncé.

Les enclos de plus grande taille possèdent des fossés larges de $0,6 \mathrm{~m}$ à $1,35 \mathrm{~m}$, et sont profonds de $0,3 \mathrm{~m}$ à $0,6 \mathrm{~m}$. Malgré des coupes stratigraphiques plus profondes, peu d'unités sédimentaires ont pu être individualisées au sein des remplissages aux faciès tout aussi uniformes que ceux des petits fossés de la première série.

Le plan de ces enclos n'est finalement jamais réellement circulaire, surtout pour les enclos de petit diamètre, qui adoptent la plupart du temps une forme ovalaire, comprenant parfois un aplatissement sur un côté, comme dans le cas des fossés 73 et 115 . On y verra un travail quelque peu intuitif, sans recours aux méthodes de mesures précises comme l'utilisation d'un cordeau par exemple.

La récurrence des petits diamètres n'est cependant pas anodine et témoigne d'une adaptation volontaire du diamètre du monument, soit en fonction de la place disponible au sein de la nécropole (alignement des 


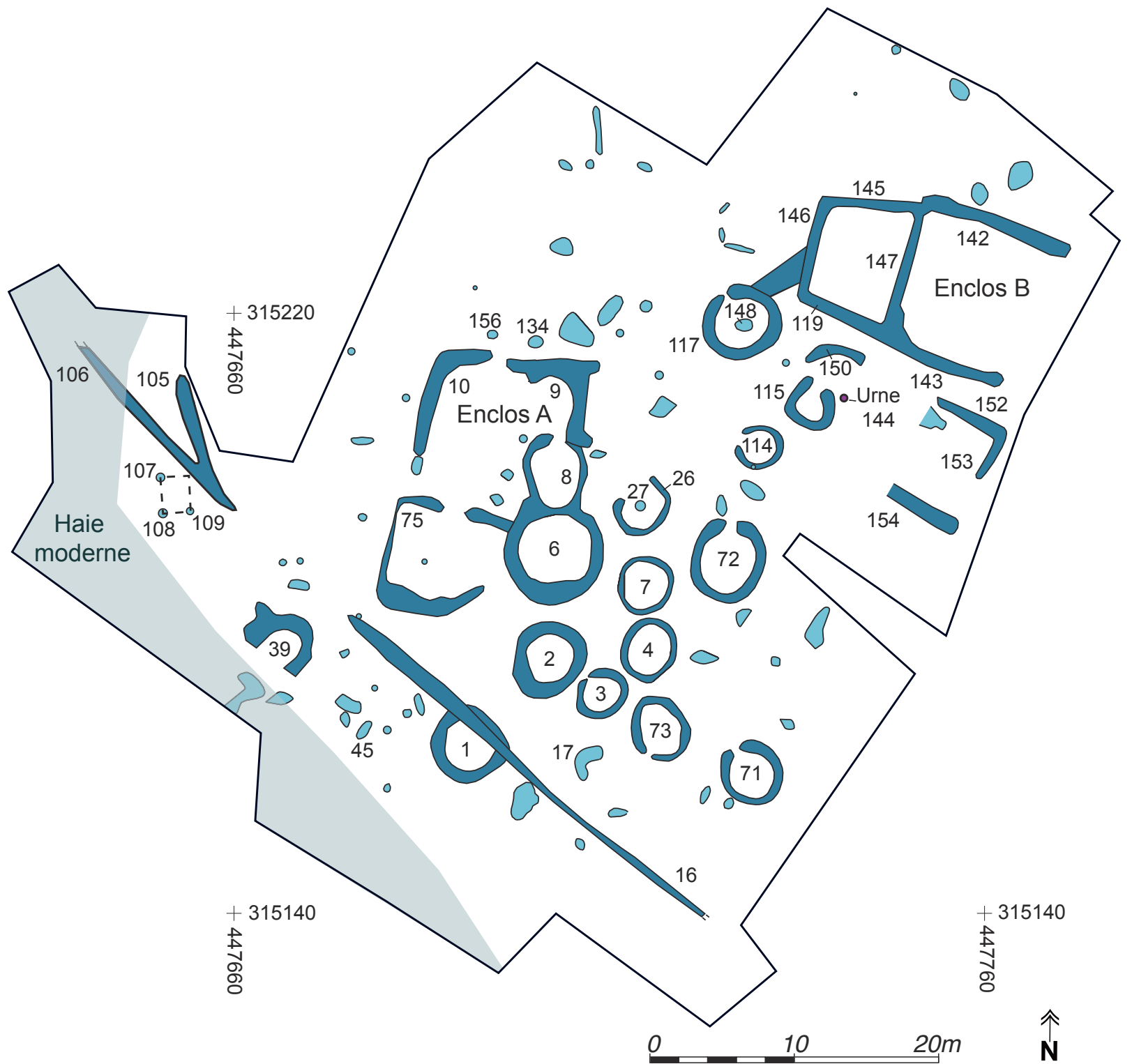

Figure 2 : Plan d'ensemble de la nécropole des Truberdières (৫ A.-L. Hamon).

Figure 2: Site plan of the Truberdières cemetery (๑ A.-L. Hamon).

enclos 73, 4, 7 et 26 par exemple), soit dans le cadre d'une transposition symbolique de la place qu'occupait le défunt au sein de la communauté. L'indigence du mobilier ici ne permet pas de préciser les positions sociales des sujets.

Les ouvertures observées sur de nombreux anneaux fossoyés sont majoritairement placées au nord (72 et 8), parfois au nord-ouest ou au nord/nord-ouest (114 et 3, 71 et 148), plus rarement au nord/nord-est (115) et au sud/sud-ouest (73). Dans le cas d'ouvertures supérieures à $0,5 \mathrm{~m}$, les interruptions des tracés peuvent matérialiser des accès à l'espace interne des enclos. Le cas des ouvertures de très petites dimensions, de l'ordre de $0,25 \mathrm{~m}$ à $0,45 \mathrm{~m}$ (numéros 3, 117 et 45), pose le problème de la fonction de cet espace trop étroit pour assurer une fonction d'entrée et renouvelle le questionnement sur la configuration d'origine de ces monuments.

Parmi les quinze enclos inventoriés sur le site, seuls les deux cercles 26 et 117 comportent une fosse centrale.

Le creusement 148, parfaitement placé au centre de l'enclos 117, est de forme circulaire. Malgré un diamètre de $1,2 \mathrm{~m}$, il n'est profond que de $0,15 \mathrm{~m}$, et comporte un remplissage hétérogène de sable brun clair à brun foncé qui n’a livré aucun mobilier.

De la même manière, la fosse 27 occupe l'espace central du petit enclos 26. Large de $0,8 \mathrm{~m}$, elle adopte un creusement en cuvette sur $0,28 \mathrm{~m}$ et n'a, elle non plus, donné lieu à aucune découverte d'objets ou d'ossements. 


\begin{tabular}{|c|c|c|c|c|c|}
\hline $\begin{array}{c}\text { Numéro } \\
\text { de fait }\end{array}$ & $\begin{array}{c}\text { Dimensions externes } \\
(\mathbf{m})\end{array}$ & $\begin{array}{c}\text { Largeur de fossé } \\
\mathbf{( m )}\end{array}$ & Profondeur $(\mathbf{m})$ & $\begin{array}{c}\text { Position } \\
\text { de l'ouverture }\end{array}$ & $\begin{array}{c}\text { Largeur } \\
\text { de l'ouverture }(\mathbf{m})\end{array}$ \\
\hline 3 & $3,8 \times 3,2$ & 0,5 à 0,65 & 0,16 à 0,33 & Nord-ouest & 0,25 \\
\hline 4 & $3,8 \times 4,5$ & 0,35 & 0,07 & Fossé fermé & $/$ \\
\hline 7 & 4 & 0,64 & 0,36 & Fossé fermé & $/$ \\
\hline 8 & $4,5 \times 5,5$ & 0,25 à 0,68 & 0,25 & Nord & 0,9 \\
\hline 26 & 4 & 0,46 à 0,52 & 0,16 à 0,27 & Incomplet? & \\
\hline 71 & $4,2 \times 4,6$ & 0,44 à 0,8 & 0,18 à 0,25 & Nord/nord-ouest & 0,56 \\
\hline 73 & $4 \times 4,8$ & 0,4 à 0,62 & 0,12 & Sud-ouest & 0,45 \\
\hline 114 & 3,2 & 0,3 à 0,48 & 0,9 à 0,17 & Ouest/nord-ouest & 0,94 \\
\hline 115 & 3,4 & 0,5 à 0,8 & 0,11 à 0,27 & Nord & 0,9 \\
\hline
\end{tabular}

Tableau 1: Dimensions des enclos de petit diamètre (৫ A.-L. Hamon).

Table 1: Dimensions of the smaller monuments (๑ A.-L. Hamon).

\begin{tabular}{|c|c|c|c|c|c|}
\hline $\begin{array}{c}\text { Numéro } \\
\text { de fait }\end{array}$ & $\begin{array}{c}\text { Dimensions externes } \\
(\mathbf{m})\end{array}$ & $\begin{array}{c}\text { Largeur de fossé } \\
(\mathbf{m})\end{array}$ & Profondeur $(\mathbf{m})$ & $\begin{array}{c}\text { Position } \\
\text { de l'ouverture }\end{array}$ & $\begin{array}{c}\text { Largeur } \\
\text { de l'ouverture }(\mathbf{m})\end{array}$ \\
\hline 1 & 5 & 1,1 & 0,62 & Fossé fermé & $/$ \\
\hline 2 & 5 & 1,1 à 1,35 & 0,55 & Fossé fermé & $/$ \\
\hline 6 & 7 & 0,95 à 1,15 & 0,48 & Fossé fermé & $/$ \\
\hline 39 & 4,8 & 1,2 & 0,42 & Incomplet & $/$ \\
\hline 72 & $5 \times 5,8$ & 0,72 à 0,95 & 0,3 à 0,57 & Nord & 0,66 \\
\hline 117 & 5,4 & 0,6 à 0,88 & 0,28 à 0,34 & Nord/nord-ouest & 0,3 \\
\hline
\end{tabular}

Tableau 2 : Dimensions des enclos de grand diamètre (৫ A.-L. Hamon).

Table 2: Dimensions of the larger monuments (๑ A.-L. Hamon).

Il est indéniable, malgré l'absence de mobilier archéologique, que ces fosses, soigneusement placées au centre des monuments, font partie intégrante de l'ensemble funéraire, voire du dispositif sépulcral. Couramment observées au sein de nombreux enclos circulaires protohistoriques, les fosses centrales livrent parfois les restes d'inhumations. À Écommoy, le caractère funéraire des fosses ne reste qu'une supposition du fait de l'absence d'indices archéologiques dans les comblements. De la même manière, l'état d'arasement prononcé du site, l'absence de caractéristiques marquées des profils et des sédiments de colmatage des deux structures ne peuvent plaider pour des restes d'ancrages de poteaux centraux.

\section{LES ENCLOS QUADRANGULAIRES}

La nécropole comporte également deux ensembles aux tracés rectilignes, dénommés $\mathrm{A}$ et $\mathrm{B}$, étroitement liés aux enclos annulaires.

Le tracé de l'ensemble A est complexe. Il regroupe quatre enclos composés des deux fossés curvilignes 6 et 8, et des fossés aux tracés rectilignes 75, 10 et 9 (fig. 3).
Aucune des intersections entre fossés n'a pu livrer d'informations concernant la relation chronologique liant les différents aménagements, cependant, la lecture de la disposition en plan des éléments de l'ensemble permet de proposer un scénario de l'installation des fossés.

Le petit enclos ovalaire 8, ouvert au nord, constitue manifestement le premier monument de la série. L'anneau 6 , le plus imposant du site, avec $7 \mathrm{~m}$ de diamètre, se greffe au sud du fossé 8 . Il est délimité par un fossé creusé en " $V$ " évasé, d' $1 \mathrm{~m}$ de large et de $0,5 \mathrm{~m}$ de profondeur. Un sondage pratiqué sur son quart sud-ouest a mis en évidence un comblement de sable meuble, brun lité de rouille.

Sur le côté occidental de l'enclos 6 prend place le fossé 75 . De largeur irrégulière, celui-ci délimite sur $8 \mathrm{~m}$ de côté un espace grossièrement carré. Un espace de $2 \mathrm{~m}$ sépare l'extrémité est du fossé 75 de l'enclos circulaire 6, tandis que sur son côté nord, figure une interruption du creusement de $1,5 \mathrm{~m}$.

Enfin, un second enclos quadrangulaire vient s'accoler au nord de cet ensemble, sous la forme des deux fossés 9 et 10 , formant chacun un angle droit. Le fossé 10 ne s'accole pas directement avec le fossé 75 , dont il est séparé 


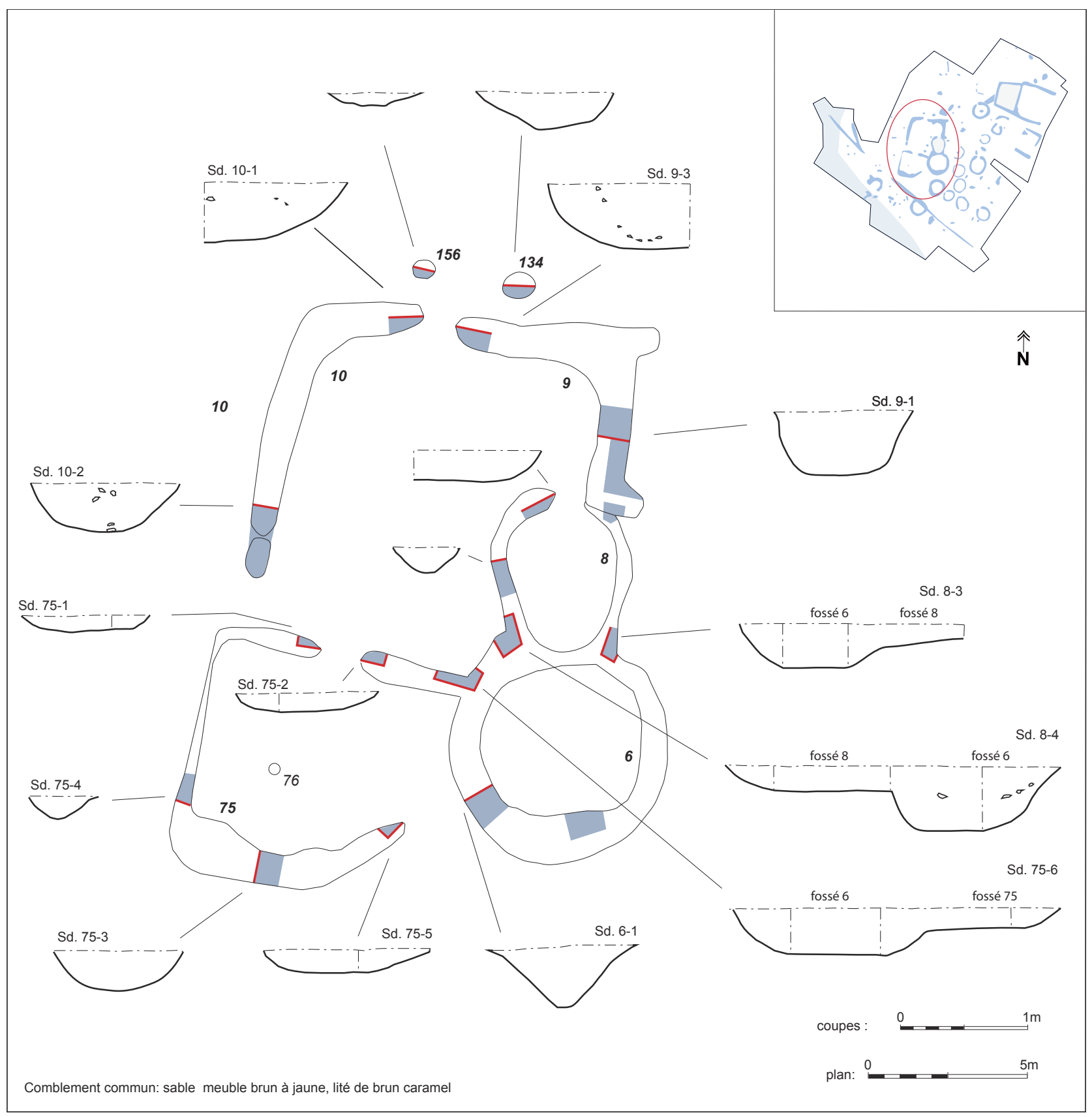

Figure 3 : L'ensemble A (๑ A.L. Hamon).

Figure 3: Site A (० A.-L. Hamon).

par 1,6 m d'espace libre. Sur la limite nord de cet enclos quadrangulaire, les deux fossés 9 et 10 ne se rejoignent pas et forment ainsi une ouverture d'1 $\mathrm{m}$ de large. À l'est, le fossé 9 se greffe sur le petit enclos annulaire 8 .

L'ensemble A se compose ainsi de quatre espaces, deux à limites curvilignes et deux de configurations quadrangulaires, le tout s'étendant sur $18 \mathrm{~m}$ de longueur et $15 \mathrm{~m}$ de largeur selon une orientation nord-nord-est/sud-sudouest. L'imbrication des différents éléments constitutifs de ce complexe funéraire implique qu'un temps relativement réduit s'est écoulé entre l'installation des enclos.
Hormis quelques esquilles d'os brûlés de nature indéterminée trouvées à $0,3 \mathrm{~m}$ de profondeur dans la branche nord du fossé 9, un lot de trois fragments de 6 ou $7 \mathrm{~cm}$ d'os non brûlés ont été retrouvés au même niveau qu'une urne renversée, à l'occasion du sondage $9 / 1$. Ces ossements se trouvent conservés grâce à leur oxydation au contact d'un objet en alliage cuivreux. Ils comprennent une portion distale d'humérus (gauche?), un fragment de diaphyse de fémur ou de tibia et le morceau proximal d'une diaphyse radiale non latéralisée. À cela, s'ajoute la couronne de la première molaire inférieure gauche. Les 
cuspides ne comportant aucune trace d'usure, il pourrait s'agir d'un adolescent si la mastication du sujet se déroulait normalement. On note la présence de matière organique sur l'humérus et le radius.

Un bracelet en bronze était en contact avec la face postérieure de l'humérus de sorte qu'il semble avoir été porté au bras plutôt qu'au poignet.

Par ailleurs, la proximité de trois types d'os, humérus (zone du coude), radius (zone du coude) et d'un ossement du membre inférieur a pu se faire de manière aléatoire, les portions d'os se trouvant après perturbations à côté du bracelet en bronze. Il est également envisageable qu'il s'agisse là d'un reliquat d'une sépulture à inhumation, les avant-bras étant fléchis sur les bras et les membres inférieurs repliés et remontés vers le thorax de sorte que l'un des coudes touche la cuisse ou la jambe.

Parmi les excavations repérées aux alentours des fossés, seules interpellent les deux fosses 134 et 156, placées au nord immédiat de l'accès nord de l'enclos septentrional de l'ensemble A. Avec respectivement des diamètres de $1,05 \mathrm{~m}$ et $0,7 \mathrm{~m}$ et des profondeurs de $0,3 \mathrm{~m}$ et $0,09 \mathrm{~m}$, ces deux structures évoquent, dans leur position, un système de portique ou d'auvent externe. La position du creusement 134, légèrement décalé vers l'est par rapport à l'ouverture du fossé, relativise cependant cette hypothèse.

Un second double enclos quadrangulaire dénommé B s'observe dans l'angle nord-est du site (fig. 4). À l'ouest, un premier espace trapézoïdal comporte deux limites fossoyées nord et sud de 7,5 m de longueur, et un côté occidental de 7,5 m également. Aux extrémités orientales de ces derniers viennent se greffer trois fossés, de $10 \mathrm{~m}$ et $11 \mathrm{~m}$ en est-ouest, et $12 \mathrm{~m}$ en nord-sud formant un espace laissé ouvert vers l'est.

Deux sondages pratiqués au niveau des intersections entre les deux enclos démontrent l'antériorité de la partie occidentale. L'ensemble a donc été créé en deux fois, un premier enclos de forme légèrement trapézoïdale ayant été creusé à l'ouest (B1), avant d'être prolongé vers l'est par les fossés de l'ensemble B2. Aucun indice ne vient démontrer l'existence d'une limite orientale primitive à l'enclos B1. Il est possible que celui-ci ait fonctionné comme l'enclos B2, sans côté est. Trois sondages effectués sur le fossé délimitant l'enclos B1 lui attribuent une largeur de $0,65 \mathrm{~m}$ à $0,8 \mathrm{~m}$, une profondeur très réduite de $0,12 \mathrm{~m}$ et un comblement homogène de sable meuble gris clair.

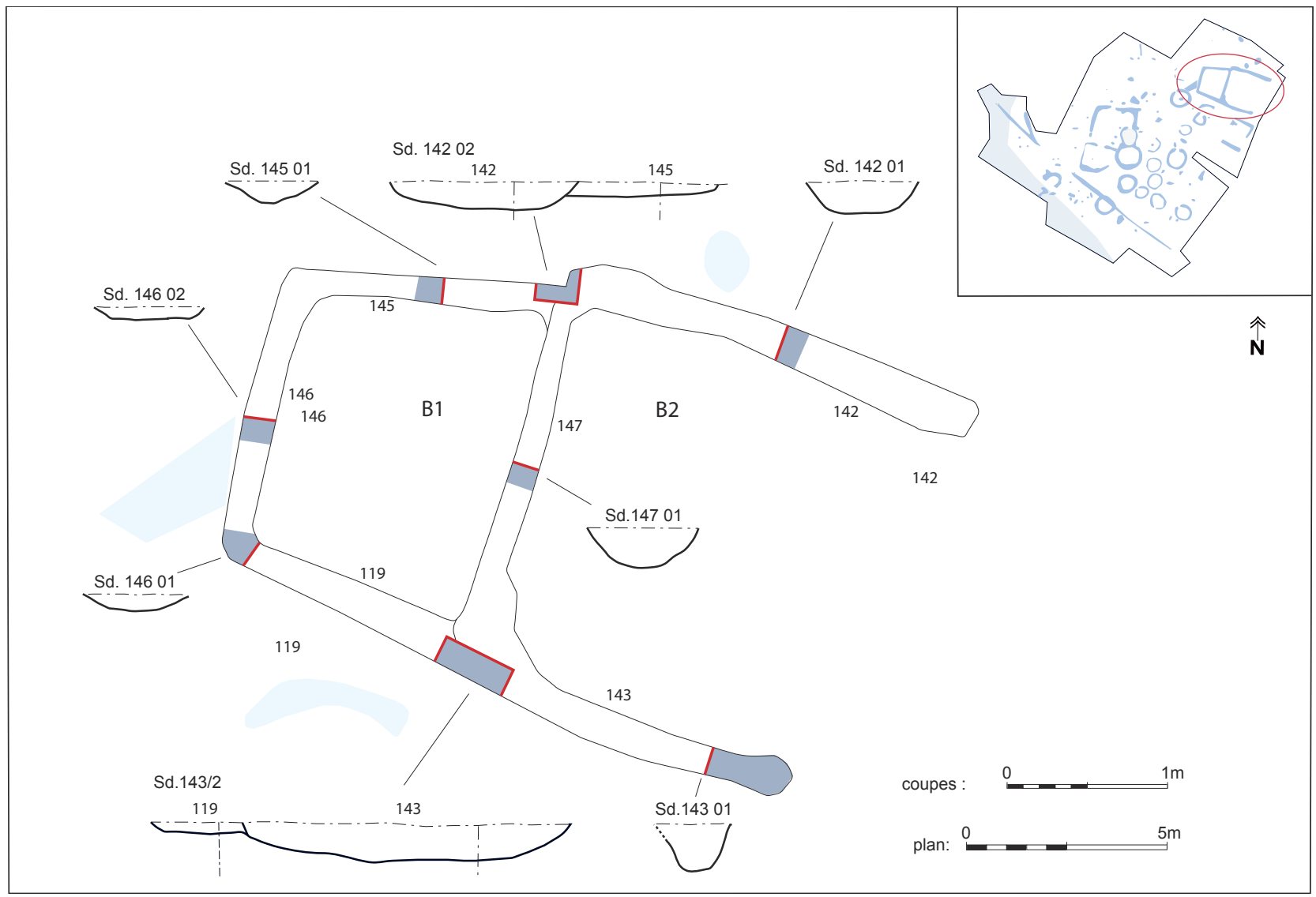

Figure 4 : L'ensemble B (C A.-L. Hamon). 
Les fossés délimitant l'enclos B2 atteignent quant à eux une largeur moyenne de $0,8 \mathrm{~m}$ et $0,3 \mathrm{~m}$ de profondeur. C'est dans cette partie de l'ensemble B, dans les sondages 143.01 et 142.03 , qu'ont été mis au jour deux petits ensembles d'os brûlés, distribués sur toute la hauteur du remplissage. En 143, il s'agit de 46,6 g d'os humains appartenant au squelette postcrânien, principalement au secteur des membres inférieurs. On trouve en effet des portions de diaphyse tibiale (23,8 $\mathrm{g}$ - portion proximale, fragments du bord antérieur de $8,9 \mathrm{~mm}$ d'épaisseur) dont un fragment de $14 \mathrm{~cm}$, déposé probablement déjà cassé en deux. Ils sont associés à un morceau du talus $(1 \mathrm{~g}-$ facette articulaire avec le tibia) et un fragment de fibula $(0,2 \mathrm{~g})$. À cela, s'ajoutent $21,6 \mathrm{~g}$ de portions d'os longs indéterminés, fortement fragmentés de $2 \mathrm{~cm}$ au plus. Bien que les fissures soient peu nombreuses, la déformation de la diaphyse tibiale courbée par la chaleur est forte. Il s'agit d'os incinérés, brûlés jusqu'à obtention d'une coloration blanche uniforme.

D'après l'épaisseur de l'os cortical du tibia, le sujet décédé serait un adulte gracile ou un grand adolescent.

Dans le fossé 142 , l'ensemble des vestiges représente $20,5 \mathrm{~g}$ de fragments, tous issus d'os longs dont 7,5 g appartiennent à des ossements épais comme le fémur ou le tibia et 2,6 gà un tibia (bord antérieur non mesurable). Les os sont complètement calcinés, de couleur blanche. Les plus grandes portions mesurent $3 \mathrm{~cm}$. D'après l'épaisseur de la partie corticale, ce reliquat de squelette correspondrait à un sujet adulte gracile ou un grand adolescent.

Les indices ténus d'un autre enclos peuvent apparaître sous la forme des fossés $150,152,153$ et 154 , placés à $2 \mathrm{~m}$ au sud et en parallèle de l'ensemble B. Sans qu'il ait été possible de relier de manière sûre ces trois tronçons fossoyés, leur forme et leur disposition évoquent les restes d'un aménagement de $15 \mathrm{~m}$ de longueur et $8 \mathrm{~m}$ de largeur. Le fossé 152/153 forme en tous les cas un angle droit pouvant correspondre au fossé 154 . Celui-ci constituerait la limite sud, et le fossé 150 l'angle nordouest d'un espace de $120 \mathrm{~m}^{2}$. Avec des largeurs de $0,8 \mathrm{~m}$ à $1,4 \mathrm{~m}$, ces fossés adoptent des gabarits légèrement supérieurs à ceux des enclos $\mathrm{A}$ et $\mathrm{B}$. Leur profondeur reste cependant très faible, de l'ordre de $0,2 \mathrm{~m}$.

\section{LE TRACÉ LINÉAIRE 105/106/16}

Le tracé 105/106/16, reconnu sur une longueur totale de $58 \mathrm{~m}$, comprenant une interruption de $11 \mathrm{~m}$ s'étend selon une orientation nord-ouest/sud-est, parallèle à l'alignement des petits enclos annulaires 2, 3, 73 et 71 dont il est distant de $6,5 \mathrm{~m}$. Ses dimensions varient de $0,75 \mathrm{~m}$ à $1,2 \mathrm{~m}$ de largeur et $0,18 \mathrm{~m}$ à $0,44 \mathrm{~m}$ de profondeur. Son tracé, réduit à $0,25 \mathrm{~m}$ de largeur sur sa partie est, correspond à un arasement prononcé du fossé au niveau des parties basses du site. Quelques restes osseux brûlés apparaissaient en surface de comblement. La découverte de deux petits anneaux en bronze en position stratigraphique profonde dans la partie occidentale du fossé 16 atteste son rattachement chronologique à la nécropole, dont il a pu constituer une limite méridionale. Dans cette perspective, on doit admettre l'existence de deux phases de fonctionnement pour l'ensemble funéraire, du fait de l'intersection du fossé 16 et du petit enclos annulaire 1, sans que l'on sache lequel des deux a précédé l'autre.

\section{LES INDICES DE BÂTIMENTS}

De nombreux fossés et trous de poteau se répartissent de manière inégale sur le site. Si la moitié d'entre eux a été sondée, bien peu ont livré quelque indice permettant de les interpréter. Hormis une concentration d'excavations de diverses tailles placées à l'ouest de l'enclos annulaire 1 , dont un seul a livré un tesson d'époque néolithique, seuls trois trous de poteau isolés dans la partie sud-ouest du site présentent un intérêt notable.

La disposition de ces trois creusements suggère le plan incomplet d'un petit bâtiment sur quatre poteaux dont le quatrième trou de fondation serait occulté par le fossé 106. Cette petite construction se compose des excavations circulaires 107,108 et 109 de $0,5 \mathrm{~m}$ de diamètre, et d'une profondeur comprise entre $0,12 \mathrm{~m}$ et $0,2 \mathrm{~m}$. Les comblements de sable homogène n'ont livré aucun mobilier et il reste impossible, pour cette construction d'un type fréquemment rencontré sur les habitats, de déterminer un lien quelconque avec la nécropole.

\section{LA SÉPULTURE À CRÉMATION 144}

Très détériorée, la sépulture 144 , située à $0,3 \mathrm{~m}$ à l'est du petit enclos annulaire 115 (fig. 1) est apparue sous la forme d'un fond d'urne en céramique contenant encore quelques ossements brûlés et une fibule en fer (cf. infra et fig. 5 et 6 ). L'ensemble osseux, de $12 \mathrm{~g}$, se compose d'une douzaine de petits fragments issus du squelette céphalique $(2,1 \mathrm{~g})$, d'une diaphyse fémorale $(6,1 \mathrm{~g})$, d'os longs indéterminés $(2,3 \mathrm{~g})$ et de $1,5 \mathrm{~g}$ d'os non identifiés. Il pourrait s'agir d'un sujet adulte d'après l'épaisseur des os longs et de la calotte crânienne.

Quelques tessons de céramique épars accompagnaient ces restes, certainement déposés dans une fosse dont les contours n'étaient plus discernables sur le substrat sableux. L'urne 144 est restée le seul témoin de ce type de sépulture sur le site. 


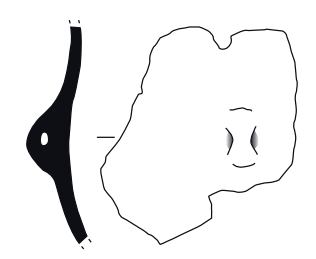

1 (F.17)

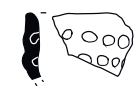

2 (F. 45)

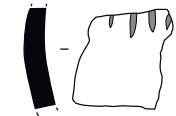

3 (F.8, sd 2)

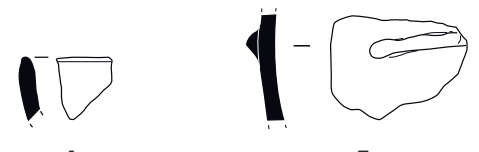

4 (F.12)

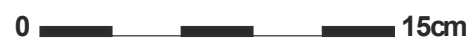

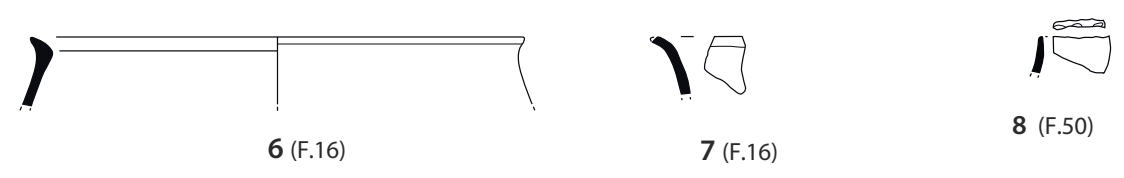

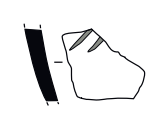

9 (F.101)

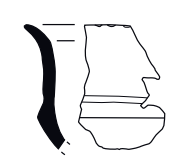

10 (F.117)

\section{CRÉAtION ET DÉVELOPPEMENT DE LA NÉCROPOLE}

Les rares objets mis au jour dans les sédiments de colmatage des fossés demeurent malgré tout suffisamment explicites pour définir l'appartenance culturelle de la nécropole et la dater. La convergence des résultats issus de l'étude céramologique et de celle des parures en métal suggère une origine et un fonctionnement du site funéraire à partir de la fin du vi ${ }^{e} s$. et au cours du v $v^{e} s$. av. n. è. Grâce à la présence de la tombe à crémation datée du $\mathrm{IV}^{\mathrm{e}} \mathrm{s}$. av. n. è., nous savons que le lieu demeure consacré aux morts sur une période de cent à deux cents ans, ce qui n'est pas exceptionnel et peut même paraître court au regard de certains sites exploités de manière continue ou intermittente sur plusieurs centaines d'années, comme la nécropole de Champ-Vallée, à Cholet (Maine-et-Loire), occupée de manière irrégulière de l'âge du Bronze ancien au milieu du second âge du Fer (Sicard et Cherel, 2015).

L'observation de la répartition des sépultures au sein de la nécropole des Truberdières traduit une organisation de

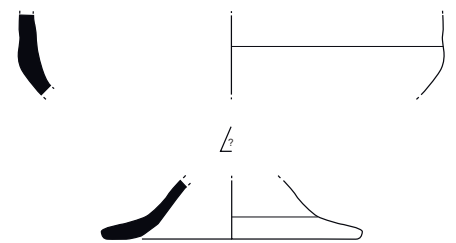

11 (F.144)

Figure 5 : Mobilier céramique du site des Truberdières (c) A. Levillayer).

Figure 5: Pottery from the Truberdières site (c) A. Levillayer).

14 (F.2, sd 1)

l'espace en fonction des types et des formats des enclos, sur une aire probablement limitée, dont $2600 \mathrm{~m}^{2}$ ont été fouillés. Elle laisse ainsi apparaître des dispositions et des associations à partir desquelles on entrevoit l'ordre chronologique d'apparition des monuments funéraires.

D'après l'étude du complexe $\mathrm{A}$, ce sont les enclos circulaires qui inaugurent le site, avec, pour éventuelle tombe fondatrice, le cercle 6 , le plus imposant des enclos annulaires, à partir duquel s'installent les anneaux 8,2 , puis les deux lignes de petits cercles 3, 73, 71, puis 4, 7 et 27 . Les deux enclos quadrangulaires $\mathrm{A} 1$ et $\mathrm{A} 2$ se greffent par la suite, peut-être sur un temps court, sur les enclos 6 et 8. Des tombes isolées s'insèrent entre ces groupes, à tout moment du fonctionnement de l'ensemble.

$\mathrm{Si}$, sur de nombreux sites comparables, les enclos curvilignes précèdent les enclos quadrangulaires, le fait de trouver, à Écommoy, un fragment de gobelet jogassien (cf. infra et fig. 5) au sein du comblement de l'un des fossés annulaires $\left(\mathrm{n}^{\circ} 117\right)$ tend à démontrer un lien de contemporanéité entre les deux types de monuments, mis en place à la transition entre le premier et le second âge du Fer. 
Seule l'urne cinéraire 144 (fig. 7) intervient tardivement, plusieurs dizaines d'années après l'installation des enclos, au cœur d'un espace funéraire sans doute déjà marqué par le temps mais dont le caractère sacré se percevait encore.
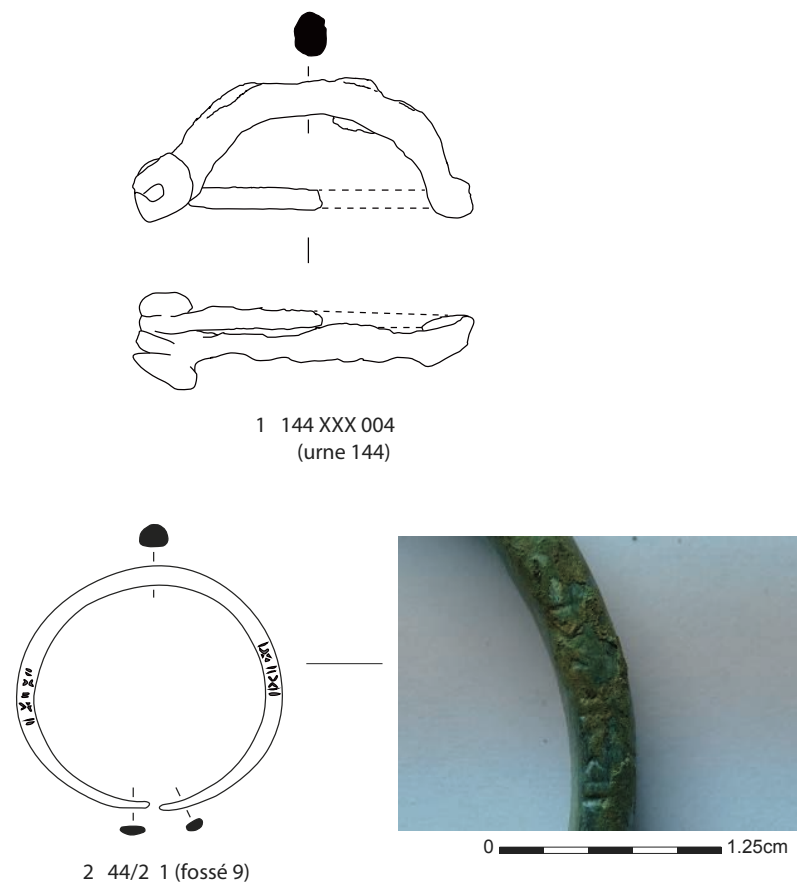

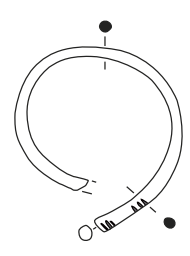

3 43/2 (fossé 16)

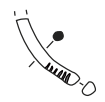

4 44/2 2 (fossé 16)

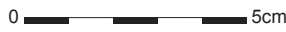

Figure 6 : Mobilier métallique du site des Truberdières (๑ A. Levillayer).

Figure 6: Metal finds from the Truberdières site (O A. Levillayer).

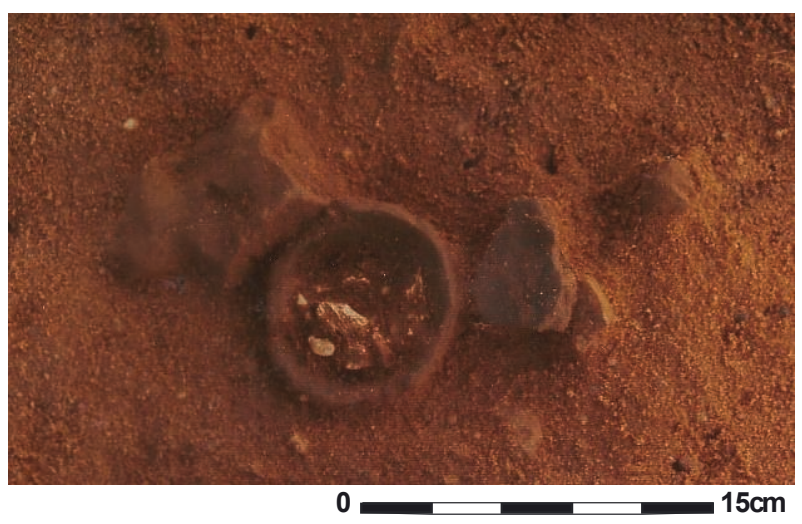

Figure 7 : L’urne cinéraire F 144 (० A.L. Hamon).

Figure 7: Cinerary urn F144 (O A.L. Hamon).
Par ailleurs, l'hypothèse est émise d'un probable glissement de la fonction des enclos fossoyés. De forme circulaire, interrompue ou non, le fossé est alors partie prenante d'un dispositif sépulcral complexe hérité de l'âge $\mathrm{du}$ Bronze qui, avec un tumulus ou un talus et une fosse centrale, constitue un monument funéraire dédié à un seul défunt. Ce monument agrège en général d'autres tombes aux architectures plus simples. En revanche, les complexes de fossés A et B relèveraient d'une autre fonction, celle de délimiter un espace cultuel ou sépulcral spécifique pouvant regrouper plusieurs défunts sans être dédié à l'un d'entre eux en particulier.

La plupart des vestiges osseux proviennent de ce contexte spécifique (fossé 9, fossé 142, fossé 143), à l'exception des os brûlés et des bracelets découverts dans le fossé rectiligne 16. Quant aux monuments funéraires circulaires, ils comportent parfois une structure centrale (fosse 148 dans l'enclos 117, fosse 27 dans l'enclos 26) qui, dépourvue de vestiges, n’apporte pas de précisions quant aux gestes funéraires. Excepté dans le fossé 115, aucun vestige osseux n'a été découvert dans les portions d'enclos circulaires fouillées.

Le dépôt d'une inhumation dans le fossé 9 et celui d'os brûlés dans les fossés des enclos quadrangulaires signalent leur possible fonction sépulcrale. Ces situations peuvent exprimer le statut de subordination du défunt ou encore marquer des secteurs stratégiques de l'espace funéraire (entrée, axe de circulation...). Les dépôts cinéraires dans les fossés 142 et 143 s'avèrent des plus sobres; dépourvus de contenant - du moins identifiable - et de mobilier, ils sont réduits à peu de chose alors que le corps de l'adolescente (?) inhumée dans le fossé 9 comportait une parure et peut-être un vase d'accompagnement. À noter que les os sont perturbés sans que l'on puisse préciser le moment de leur remaniement (sont-ils en position primaire ou s'agitil d'un dépôt secondaire?).

Le contexte de l'inhumation est compris entre le début du v et la fin du Iv $v^{e}$ s. av. n. è., tandis que celui des crémations dans les fossés 142 et 143 demeure non daté.

La tombe 144 atteste la pratique de la crémation dès le IV ${ }^{e}$ s. à Écommoy et confirme son usage précoce dans les Pays de la Loire et en Bretagne (Dedet et Le Goff, 2018). Dans ces régions, cette tendance est mise en place au cours du ve ${ }^{\mathrm{e}}$ s. et perdure jusqu'au $\mathrm{III}^{\mathrm{e}} \mathrm{s}$. (Villard-Le Tiec et al., 2010). Ce dépôt cinéraire, plus élaboré que ceux mis au jour dans les fossés 142 et 143, est inséré dans un contenant, et placé dans une tombe propre au défunt, éventuellement associée à l'enclos 115. Par ailleurs, une fibule figure parmi les restes osseux.

Malgré la rareté des restes osseux conservés, le traitement des défunts sur ce site pose la question de la fonction sépulcrale des fossés quadrangulaires voués par 
ailleurs à la délimitation des espaces funéraires. Le site illustre également les modalités d'usage de la crémation $\mathrm{au} \mathrm{IV}^{\mathrm{e}} \mathrm{s}$. ainsi que la relation entre la pratique crématoire et celle de l'inhumation.

\section{LE MOBILIER CÉRAMIQUE (A. LEVILLAYER)}

Malgré un décapage et une fouille assez poussés, le site des Truberdières livre un corpus assez réduit, avec un total de 244 tessons (poids : 1,455 kg), parmi lesquels on recense 14 éléments de forme, dont 8 individus (fig. 5). À l'exception de quelques vases, il s'agit de tessons de petite taille, très fragmentés et dont l'état de conservation est mauvais. Les surfaces sont altérées et la lecture des traitements de surface très difficile. Malgré tout, certains objets remarquables et leur association avec des éléments de parure font de cet ensemble un lot tout à fait intéressant à l'échelle régionale. Une vingtaine d'objets en silex, dont deux petits grattoirs ont été retrouvés au cours de la fouille, souvent en accompagnement de la poterie, au sein des comblements.

En préambule, il convient de signaler que plusieurs fragments de poterie néolithiques ont été découverts sur le site (fig. 5, nos 1 à 3 ). Outre leurs caractéristiques typologiques, ils présentent des pâtes différentes de celles rencontrées sur les céramiques protohistoriques. On signalera essentiellement la découverte d'une panse ovoïde à anse à œeillet dans la fosse 17 et d'un bord à décor pastillé réalisé « au repoussé » dans la fosse 45. Ces éléments semblent renvoyer au Ve millénaire (Cassen et al., 1999).

Un fragment de panse présente un cordon lisse rapporté qui semble légèrement courbé (arciforme?). La panse présente un profil très peu marqué mais est trop incomplète pour autoriser une datation précise (fig. $5, \mathrm{n}^{\circ} 5$ ). Toutefois, ce tesson découvert dans la fosse 12 (non localisée sur le plan général), associé à un petit bord aminci (fig. $5, \mathrm{n}^{\circ} 4$ ), évoque le Bronze ancien (Brunet et al., 2008).

Le fossé 16 a livré 17 tessons, parmi lesquels deux éléments de bords (fig. $5, n^{\text {os }} 6$ et 7 ). Une lèvre éversée à méplat interne est trop fragmentaire pour pouvoir être caractérisée, mais elle semble participer d'un haut col. Le vase $n^{\circ} 6$ en revanche présente un col atrophié à lèvre à méplat effilée légèrement rentrante, qui correspond probablement à un pot à panse ovoïde. La pâte fine légèrement micacée est soigneusement lissée. Cet élément renvoie à des formes connues dans les deux seuls ensembles mayennais datés de la fin du premier âge du Fer, le site du Château à Sainte-Suzanne (étude C. Marcigny dans : Bocquet, 2008, p. 15-16) et celui de La Hennerie à Argentré (étude A. Levillayer dans : Morin, 2015, p. 46-55), qui corrèlent à d'autres ensembles contem-
2009, fig. 16, $\mathrm{n}^{\text {os }} 2-3$ ), encore que ce type de lèvre est en germe dès le début du premier âge du Fer (Levillayer, 2013, fig. 4, no 18). La découverte d'éléments annulaires en alliage cuivreux dans ce fossé conforte une datation à la fin du premier âge du Fer ou au début de la période suivante (cf. infra et fig. 6).

Le fossé circulaire 117 a pour sa part livré l'élément le plus caractéristique du corpus (fig. 5, $\mathrm{n}^{\circ} 10$ ). Il s'agit d'une écuelle à panse carénée et col haut légèrement concave terminée par une lèvre déjetée. La pâte micacée a été cuite en atmosphère réductrice contrôlée lui donnant une couleur noire uniforme. Les surfaces ont été soigneusement lissées, attestant d'une attention particulière portée à la fabrication du vase. Cette forme, récurrente dans les deux ensembles mayennais déjà cités, s'insère dans les formes associées aux "gobelets jogassiens ", de mieux en mieux identifiées dans l'Ouest, et dans les Pays de la Loire notamment (Cornec et al., 2018, forme 12b). Elle autorise une datation centrée sur la première moitié du v $v^{e} s$. av. n. è. et annonce La Tène ancienne.

L'urne 144 (fig. 5, no 11) est particulièrement intéressante dans la mesure où sa fonction funéraire est assurée et que, outre des ossements incinérés, elle contenait une fibule en fer (cf. infra et fig. 6). Le vase est représenté par son pied et une partie de la panse, la jonction entre les deux n'ayant pu être restituée. Cette céramique modelée est très abîmée. On remarque toutefois un lissage des surfaces à la spatule. Son pied creux relativement haut, de type "piédouche", supporte une probable panse carénée (à col subcylindrique?). La découverte d'urnes et de coupes à piédestal est relativement fréquente dans les rares contextes funéraires régionaux, à l'image du site de Champ Vallée à Cholet dans le Maine-et-Loire (Sicard et Cherel, 2015, fig. 10). La forme très particulière du pied d'Écommoy et la morphologie générale du vase renvoient également à des vases bien documentés dans le CentreOuest pour le $\mathrm{IV}^{\mathrm{e}} \mathrm{s}$. av. n. è., notamment en contextes funéraires à Mazerolles (Vienne) ou dans la grotte des Perrats à Agris (Charente-Maritime), entre La Tène B1-B2 et La Tène B2-C1 (Gomez de Soto et al., 2007, fig. 8 et 9). Cette datation corrèle parfaitement celle de la fibule que contenait l'urne (cf. infra et fig. 6).

Le vase complet découvert dans le fossé 9 (enclos A2) interprété comme un mobilier d'accompagnement d'un défunt inhumé (Hamon, 2001, p. 32), est relativement ubiquiste. Il s'agit d'une jatte tronconique modelée à pâte sableuse à lèvre digitée (fig. $5, \mathrm{n}^{\circ} 12$ ). Cette forme abonde dans les contextes régionaux depuis le $\mathrm{VI}^{\mathrm{e}} \mathrm{s}$. jusqu'à la fin de la période, et ne peut pas constituer un indicateur chronologique. La lèvre digitée pourrait toutefois la placer dans une fourchette haute, entre la fin du premier âge du Fer et le début du second (Cornec et al., 2018, forme 1), 
de nombreux contextes de La Tène ancienne livrant des vases similaires. Son association potentielle avec un bracelet en alliage cuivreux permettrait de confirmer une datation haute.

Les autres éléments dessinés (fig. $5, n^{\circ s} 8,9,13$ et 14 ) permettent tout au mieux une datation des structures à l'âge du Fer.

En conclusion, on constate que les éléments disponibles placent la fréquentation du site entre la fin du premier âge du Fer et La Tène ancienne, soit aux $v^{e}$ et $I v^{e} s$. av. n. è., bien qu'une fondation, peut-être dès l'âge du Bronze ou dans la première moitié du premier âge du Fer, puisse être envisagée au regard de quelques rares tessons. La présence de mobilier métallique associée à la céramique fournit des éléments rarement perceptibles dans la région.

\section{LE MOBILIER MÉTALLIQUE (C. MOREAU)}

Le site d'Écommoy-Les Truberdières a livré un mobilier métallique réduit de quatre objets en alliage cuivreux et en fer (tabl. 3). Les objets présentent un état de conservation médiocre. La masse globale, indicative en raison d'une corrosion importante, avoisine les $120 \mathrm{~g}$. La nature acide des sols armoricains ne favorise généralement pas la conservation des éléments métalliques, qui parviennent souvent dégradés et peu identifiables. Les contextes d'incinération offrent généralement de meilleures conditions de conservation, pouvant permettre une approche de la composition des dépôts funéraires.

La fibule est issue d'une urne cinéraire isolée, mise au jour au nord-est de l'emprise à proximité de l'enclos 115 (F 144). L'objet était en contact direct avec les restes incinérés d'un sujet adulte. L'arc massif présente une section lenticulaire, élargie dans sa partie médiane. Le ressort est court, à deux fois deux spires et corde externe (fig. 6, $\mathrm{n}^{\circ} 1$ ). Aucun décor n'est visible. L'arc, bas et allongé, montre un profil arrondi qui s'éloigne des arcs en demi-cintre connus pour la période hallstattienne - La Tène ancienne précoce. Le pied n'est pas conservé, mais celui-ci est généralement coudé. Cet exemplaire se rapproche par ses caractéristiques des fibules à arc tendu du IV ${ }^{e}$ s. av. n. è. et peut être mis en parallèle avec le type 313 de la séquence Aisne-
Marne IIIA (Demoule, 1999). Il correspond par ailleurs à la description des fibules de type FA20 du Bassin parisien (Marion, 2004) orientant la datation vers La Tène A2-B1. Les exemplaires de ce type restent extrêmement rares dans la région, qui plus est souvent très mal conservés.

Le bracelet issu du fossé 9 de l'enclos A2 (sondage 9/1) présente un fragment osseux fixé par minéralisation (face postérieure d'un humérus). L'hypothèse d'un reliquat d'inhumation est envisagée, accréditée par la présence d'une dent dans le sondage voisin, à une soixantaine de centimètres de l'objet. Le bracelet paraît intégrer, ainsi qu'une céramique découverte à la même altitude, le dépôt funéraire accompagnant le défunt (cf. supra). Il présente un cintrage ovale et se rapporte au type de bracelet ouvert à jonc plein lisse de section planoconvexe (fig. 6, $\mathrm{n}^{\circ} 2$ ). Les extrémités sont simples, juste sectionnées et amincies. Les éléments d'un décor incisé sont perceptibles en deux endroits. Le motif est constitué de la répétition de deux incisions annulaires partielles encadrant un motif de chevrons opposés, formant une croix. La corrosion importante rend la lecture de l'organisation du décor difficile. Néanmoins, le motif en lui-même suggère plutôt une organisation ternaire. Il présente des similitudes avec le type 252 (Demoule, 1999), renvoyant à une phase précoce de La Tène (Aisne-Marne II, potentiellement IIA), et montre également des correspondances évidentes avec le type BB12d (variante décorée) du Bassin parisien (Marion, 2004).

Deux autres bracelets ( $43 / 2$ et 44/2) ont été mis au jour dans le remplissage du fossé $\mathrm{F} 16$, entre les sondages $16 / 2$ et 16/3. Quelques ossements brûlés ont été recueillis un peu plus à l'ouest en surface du fossé, au niveau de son intersection avec le cercle fossoyé 1 , sans lien avéré avec le mobilier métallique (Hamon, 2001, p. 42).

Le premier bracelet est à jonc lisse de section circulaire et décor incisé (fig. $6, \mathrm{n}^{\circ} 3$ ). Son cintrage ovale montre une certaine déformation, sans doute liée à un ajustement. Les extrémités sont simples, soulignées par des incisions annulaires par groupes de trois pour une des extrémités (43/2), par six pour l'autre (44/2). La corrosion empêche la lecture d'un éventuel décor. Là encore, il peut être mis en parallèle avec le type 252 de la séquence Aisne-Marne

\begin{tabular}{|c|c|c|c|c|c|c|}
\hline No inventaire & Provenance & Catégorie & objet & matériau & NR & NMI \\
\hline 144_XXX_004 & Urne 144 & accessoire vestimentaire & fibule & fer & 9 & 1 \\
\hline $44 / 21$ & Fossé 9 sd. 1 & parure & bracelet & all. cu. & 1 & 1 \\
\hline $44 / 22$ & Fossé 16 & parure & bracelet & all. cu. & 6 & 1 \\
\hline $43 / 2$ & Fossé 16 & parure & bracelet & all. cu. & 4 & 1 \\
\hline
\end{tabular}

Tableau 3 : Inventaire du mobilier métallique (๑ C. Moreau).

Table 3: Inventory of metal objects (C C. Moreau). 
précoce (potentiellement IIA; dans Demoule, 1999), et correspond par ailleurs à la description du type BB12d du Bassin parisien (Marion, 2004). Le second bracelet présente globalement les mêmes caractéristiques, jonc lisse de section circulaire et décor incisé (fig. 6, no 4). L'une des extrémités conservées montre un groupe d'incisions lâches (annulaires?). L'état fragmentaire de l'objet ne permet pas de préciser ses dimensions. Néanmoins, son association avec l'exemplaire précédent de même facture, suggère une typologie similaire.

Le caractère lacunaire des données mises au jour à Écommoy ne permet pas d'appréhender précisément les modalités des dépôts funéraires. Quelques remarques peuvent néanmoins être formulées concernant les dépôts issus de la fosse 144 et du fossé 9 . La fibule du dépôt 144 présente un taux de fragmentation quasi nul. La fibule n'apparaît pas brisée volontairement, elle était peut-être portée par le défunt, puis ramassée et déposée au contact des restes osseux. Étant donné l'état de conservation très lacunaire de la structure 144 , et le fait de ne disposer que d'un seul objet, on ne peut préciser si une sélection, tout ou partie de la dotation, a été opérée au moment de rassembler les vestiges de la crémation. La composition du dépôt ne montre pas d'organisation ou de mise en scène spécifique. Le dépôt du fossé 9 , duquel est issu le bracelet en alliage cuivreux 2, est un contexte peu fiable et partiel. Pour autant, on observe que le bracelet était porté au poignet ou à l'avant-bras. Les restes du dépôt osseux mis au jour dans le fossé 9 ont permis d'identifier un sujet adolescent, sans plus de précisions. Le caractère lacunaire du dépôt et l'absence d'accessoires vestimentaires ne permettent pas de préciser une appartenance possible à la sphère féminine ou masculine.

Le mobilier métallique participe par ailleurs à l'évaluation des degrés de richesse des individus. L'importance du mobilier est fonction non seulement du nombre d'objets, mais également de sa valeur par le matériau utilisé et sa rareté. Si le site d'Écommoy ne livre pas suffisamment d'indices par sa médiocre conservation et la rareté du mobilier mis au jour, la présence de mobilier, notamment d'un bracelet en alliage cuivreux, traduit l'appartenance à un certain statut social des défunts dont les restes ont pu être fouillés.

Le corpus présenté ici renvoie principalement à une séquence La Tène ancienne précoce ( $v^{\mathrm{e}} \mathrm{s}$. av. n. è.). Les dépôts issus des fossés 9 et 16 appartiennent à une séquence relativement haute (soit fin $\mathrm{VI}^{\mathrm{e}} \mathrm{s}$. av. n. è., début $\mathrm{V}^{\mathrm{e}} \mathrm{s}$. av. n. è.). La fibule en fer s'avère potentiellement plus récente ( $\mathrm{IV}^{\mathrm{e}} \mathrm{s}$. av. n. è.), montrant une certaine hétérogénéité, somme toute relative. Les contextes sont partiels et ne permettent pas de proposer une analyse typochronologique et sociologique fine. Il est par ailleurs délicat de vouloir proposer une datation plus précise, au regard notamment du peu de références régionales. Quelques nécropoles à enclos sont connues, mais peu ont livré du mobilier métallique et souvent de manière indigente, à l'image de la nécropole de Champ Vallée à Cholet dans le Maine-et-Loire (Sicard et Cherel, 2015) ou de Meslay-duMaine en Mayenne (Morin, 2010) pour les exemples les plus proches. La nécropole de Cherré, à Aubigné-Racan (Sarthe), située dans un périmètre proche, présente à travers le mobilier du tumulus $\mathrm{n}^{\circ} 2$ un horizon précoce. Les fibules sont associées à des anneaux en fer, une tête d'épingle en fer, une tige avec crochet en alliage cuivreux, une bague en alliage cuivreux, un bracelet en pâte de verre et un collier (ou bracelet?) composé de perles (ambre et pâte de verre). Ces dernières se rapportent à la première moitié du ve s. av. J.-C. (Lambert et Rioufreyt, 1979, p. 20 ; Bouvet, 2001, p. 144-145). Cet exemple souligne une fois encore la rareté des bracelets en métal (Cornec et al., 2018).

Au final, le mobilier métallique mis au jour à Écommoy montre, par ses caractéristiques chronotypologiques, une appartenance aux domaines du Bassin parisien et de l'Aisne-Marne, et témoigne des pratiques funéraires du Nord de la France. Bien que partiel tant en contexte qu'en dépôt, il offre un aperçu particulièrement intéressant des pratiques funéraires locales au début de La Tène.

\section{MISE EN PERSPECTIVE RÉGIONALE DE LA NÉCROPOLE D'ÉCOMMOY}

Malgré l'ancienneté de la fouille et l'exiguïté du site, la petite nécropole des Truberdières demeure l'un des jalons importants d'un contexte funéraire ligérien encore peu fouillé pour la fin du premier âge du Fer.

Dans sa configuration, Écommoy peut être comparé au site de Cholet-Le Champ Vallée (Maine-et-Loire), fouillé en 2008 par S. Sicard. Sur la partie de cette nécropole occupée durant le premier âge du Fer, on retrouve le même mode d'accolement des enclos les uns sur les autres et la mixité des formes rectilignes et curvilignes.

La fin de la période hallstattienne voit également apparaître les nécropoles de Cherré à Aubigné Racan (Sarthe) [Bouvet, 2001], Aubigny-Le Baillargeau (Vendée) [Poissonnier et al., 2008] ou encore Rezé-La Brosse, la Forêt 3 (Loire-Atlantique) [Sélèque, 2012] où se rencontrent également à la fois des enclos circulaires et quadrangulaires.

La synthèse rédigée en 2009 à propos du geste funéraire rencontré à l'âge du Fer dans le Grand Ouest, (Villard-Le Tiec et al., 2010) montre la multiplicité des formes de traitements des défunts, mettant en œuvre l'inhumation et la crémation de manière alternée ou 
concomitante, dans des contextes très variables : enclos, tumulus, grottes, en urnes, en coffres, en fosses, des procédés divers souvent rencontrés sur un même site.

L'étude réalisée sur les enclos circulaires de Bretagne et des Pays de la Loire par Mickael Le Maire en 2014 puis en 2015 décèlent, à partir d'une première typologie des formes, la détermination géographique de groupes d'enclos aux formes définies (Le Maire, 2015). Avec une variété notable de plans, du fossé continu au fer à cheval, en passant par les tracés ouverts, la nécropole d'Écommoy regroupe plusieurs des types déterminés par cet auteur. Elle se distingue par ailleurs des autres séries fouillées dans la région, par la taille réduite des cercles, notamment ceux de la première série, dont les diamètres moyens n'atteignent que de 3 à $5 \mathrm{~m}$. Ces dimensions ne sont cependant pas exceptionnelles, puisque selon M. Le Maire, 29 \% des enclos inventoriés en Pays de la Loire mesurent moins de $5 \mathrm{~m}$ de diamètre.

À Écommoy, les enclos quadrangulaires qui succèdent de peu aux formes curvilignes soulignent la mixité des pratiques funéraires mises en œuvre. Enfin, l'urne cinéraire 144, bien qu'isolée dans le temps, indique la perduration du caractère funéraire et sacré des lieux, sur plus d'un siècle au-delà de la création des premières tombes.

\section{Bibliographie}

Bocquet A. (dir.), 2008, Sainte-Suzanne. Le château; extension du logis - 53.255.0001 (Campagne de fouilles de juin à septembre 2006), rapport final d'opération de fouille archéologique préventive, Nantes, Service Archéologique de la Mayenne-SRA Pays de la Loire, 2 vol., 99 p.

Bouvet J.-P., avec la coll. de Aubin G., Colin A., DesChamps S. et de Saulce A., 2001, La Sarthe, Paris, Académie des Inscriptions et Belles Lettres, Carte archéologique de la Gaule, 72, 519 p.

Brunet P., Cottiaux R., Hamon T., Magne P., Richard G., Salanova L. et Samzun A., 2008, "Les ensembles céramiques de la fin du III ${ }^{\mathrm{e}}$ millénaire (2300-1900 av. n. è.) dans le Centre-Nord de la France ", Bulletin de la Société préhistorique française, t. 105, n 3, p. 595-615.

Cassen S., Marchand G., Menanteau L., Poissonnier B., Cadot R. et Viau Y., 1999, " Néolithisation de la France de l'Ouest. Témoignages Villeneuve-Saint-Germain, Cerny et Chambon sur la Loire angevine ", Gallia Préhistoire, vol. 41, no 1 , p. 223-248.

Cornec J., Le Goff E., Le Guévellou R., Levillayer A., Moreau C., Mortreau M. et Thébaud S., 2018, " La céramique du $\mathrm{VI}^{\mathrm{e}}$ au $\mathrm{I}^{\mathrm{er}} \mathrm{s}$. av. n. è. en Pays de la Loire : évolution des formes, des décors et mobiliers associés ", dans Menez Y. (dir.), Céramiques gauloises d'Armorique. Les des- siner, les caractériser, les dater, Rennes, Presses universitaire de Rennes, coll. "Archéologie et Culture », p. 357-450.

Dedet B. et Le Goff I., 2018, "Regards sur les coutumes funéraires " ordinaires " à l'âge du Fer en France ", dans Guilaine J. et Garcia D., La Protohistoire de la France, Paris, Hermann éditeurs, coll. " Histoire et archéologie ".

Demoule J.-P., 1999, Chronologie et société dans les nécropoles celtiques de la culture Aisne-Marne du VI au III s. av. n. è., numéro spécial de la Revue archéologique de Picardie, 15, $406 \mathrm{p}$.

Gomez de Soto J. (coord.), Lejars T., Ducongé S., RoBIN K., Sireix C. et ZéLIe B., 2007, « Du milieu du vªu $\mathrm{III}^{\mathrm{e}}$ s. av. n. è. en Centre-Ouest, Aquitaine septentrionale et ouest du Massif Central ", dans Mennessier-Jounnnet C., Adam A.-M. et Milcent P.-Y. (éd.), La Gaule dans son contexte européen aux IV et III s. av. n. è., Actes du XXVII colloque de l'AFEAF (Clermont-Ferrand, 2003), Lattes, Association pour le Développement de l'Archéologie en Languedoc-Roussillon, p. 69-89.

Hamon A.-L., 2001, Écommoy Zac des Truberdières (72), DFS de fouille archéologique, Nantes, AFAN-SRA Pays de la Loire, septembre, $60 \mathrm{p}$.

LAmbert C. et Rioufreyt J., 1979, Le théâtre gallo-romain de Cherré, Aubigné-Racan, Sarthe, rapport de fouilles, Circonscription des Antiquités Historiques des Pays de la Loire.

Le Maire M., 2015, "Les enclos circulaires dans le NordOuest de la France, regard croisé entre les monuments de Bretagne et des Pays de la Loire ", dans Boulud-Gazo S. (coord.), Rapport d'activité du Projet Collectif de Recherche 2015, Le Campaniforme et l'âge du Bronze dans les Pays de la Loire, Nantes, université de Nantes-SRA Pays de la Loire, p. 81-91.

LeVillayer A., 2013, "Un ensemble du tout premier âge du Fer à Vouillé-les-Marais (Vendée) ", Bulletin du Groupe Vendéen d'Études Préhistoriques, 49, p. 15-26.

Maitay C. et Marchadier É., avec la coll. de Béhague B., 2009, «Entre traditions locales et apports exogènes : évolution et singularités de la céramique du premier âge du Fer et de La Tène ancienne entre Loire et Dordogne ", dans Bertrand I., Duval A., Gomez De Soto J. et Maguer P. (dir.), Les Gaulois entre Loire et Dordogne, Actes du XXXIe colloque de l'AFEAF (Chauvigny, 2008), Chauvigny, Association des Publications Chauvinoises, coll. "Mémoires ", XXXIV, p. 307-340.

Marion S., 2004, Recherches sur l'âge du Fer en Île-de-France. Entre Hallstatt final et La Tène finale. Analyse des sites fouillés. Chronologie et société, Oxford, BAR Publishing, International Series, 1231, 2 vol.

Moreau C. et Saurel M., à paraître, "Les mobiliers funéraires - équipement et dépôts ", dans BonNABel L. (dir.), Pratiques funéraires et sociétés de l'âge du Fer en ChampagneArdenne (Hallstatt final-La Tène moyenne). 
Morin S., avec la coll. de Paillot E. et Bouvet J.-P., 2010, Meslay-du-Maine "Moulin à Vent no 2 ", rapport final d'opération de diagnostic archéologique, Nantes, Service archéologique de la Mayenne-SRA Pays de la Loire, 43 p.

Morin S. (dir.), 2015, Argentré "La Verrerie de Gripouce " (région Pays de la Loire-Mayenne), rapport final d'opération de diagnostic archéologique, Nantes, Service archéologique de la Mayenne-SRA Pays de la Loire, 342 p.

Poissonnier B. (dir), Савот E., Marti F. et Poirier P., 2008, Aubigny "le Baillargeau ", rapport final de fouille archéologique, Nantes, Inrap-SRA Pays de la Loire, 169 p.

Sélèque J., avec la coll. de Boret B., Harster M., Leray S., Levillayer A., Scaon C. et Mens E., 2012, Rezé (44), ZAC de la Brosse - La Forêt 3, rapport final d'opération de fouille préventive, Nantes, ArchéoLoire - SRA Pays de la Loire, 120 p.

Sicard S. et Cherel A.-F., 2015, « Le vaste ensemble protohistorique de Champ Vallée à Cholet (Maine-et-Loire) : une vocation funéraire et rituelle? ", Bulletin de l'APRAB, 13, p. 107-122.

Villard-Le Tiec A., Gomez de Soto J., Bouvet J.-P. (coord.), Baranger A., Bizien-Jaglin C., Boulestin B., Dubillot X., Hamon A.-L., Kerouanton I., Landreau G., Le Bihan J.-P., Le Goff E., Le Meur N., Levillayer A., Maguer P., Mortreau M., Pautreau J.-P., Sicard S. et Villard J.-F., 2010, " Pratiques funéraires du second âge du Fer en Gaule de l'Ouest (Bretagne, Pays de la Loire, Poitou-Charentes) ", dans Barral P., Dedet B., Delrieu F., Giraud P., Le Goff I., Marion S. et Villard-Le Tiec A. (dir.), Gestes funéraires en Gaule au second âge du fer, Actes du XXXIII colloque international de l'AFEAF (Caen, 20-24 mai 2009), Besançon, Presses universitaires de Franche-Comté, Annales littéraires, 883, série «Environnement, sociétés et archéologie ", 14, p. 85-106.

Die eisenzeitliche Nekropole von Les Truberdières bei Écommoy (Sarthe)

Das Gräberfeld mit Grabeinhegungen von Ecommoy, das im Südteil des Departments Sarthe liegt, ist 2001 ausgegraben worden. Mit einer Serie von 15 kleinen Kreisgräben sowie zwei doppelten rechteckige Grabeinhegungen, die an den Übergang zwischen der älteren und der jüngeren Eisenzeit $z u$ datieren sind, handelt es sich um eines der am dichtesten belegten Gräberfelder dieser Epoche in der Region Pays de la Loire. Feine Grabbeigaben aus Keramik und Metall unterstreichen die die besondere Stellung des Gräberfeldes, das in einer Zone mit kulturellen Einflüssen aus dem Pariser Becken und der Aisne-Marne Region liegt.

Schlagwortindex: Gräberfeld, Eisenzeit, Kreisgräben, rechteckige Grabeinhegungen, Brandbestattung.

\title{
La necrópolis de la Edad del Hierro de Les Truberdières, en Écommoy (Sarthe)
}

\begin{abstract}
La necrópolis de Les Truberdières en Ecommoy, situada en el sur del departamento de la Sarthe, fue excavada en 2001. Con quince pequeños recintos circulares delimitados por fosos y dos dobles recintos cuadrados, fechados en la transición entre la Primera y la Segunda Edad del Hierro, se trata de uno de los espacios funerarios más densamente ocupados de esta época descubiertos en los Países del Loira. El escaso material cerámico y metálico contribuye a resaltar su carácter destacado, encuadrándola en el marco de la zona de influencia de la cuenca parisina y el ámbito Aisne-Marne.
\end{abstract}

Palabras clave: necrópolis, Edad del Hierro, recinto circular, recinto cuadrado, incineración. 